\title{
The Expression and Functional Significance of Vascular Endothelial-Cadherin, CD44, and Vimentin in Oral Squamous Cell Carcinoma
}

\author{
Soussan Irani ${ }^{1}$, Arash Dehghan ${ }^{2}$
}

${ }^{1}$ Department of Oral Pathology, Dental Research Centre, Research Centre for Molecular Medicine, Dental Faculty, Hamadan University of Medical Sciences,

${ }^{2}$ Department of Pathology, Besat Hospital, Hamadan University of Medical Sciences, Hamadan, Iran
Received : 15-11-17.

Accepted : 15-01-18.

Published : 24-04-18.
Objectives: Ninety percent of head and neck cancers are squamous cell carcinoma which develops in the oral cavity. Metastasis is the main causative factor for death in $90 \%$ of all cancer-related deaths and begins with the invasion of tumor cells through the walls of small blood vessels or lymph vessels. A growing body of evidence has shown that vasculogenic mimicry (VM) facilitates tumor growth and cancer metastasis. The current study aimed to present the role of vascular endothelial (VE)-cadherin, CD44, and vimentin in inducing VM and epithelial-mesenchymal transition (EMT) and to identify the cancer stem cell (CSC) niche in different grades of oral squamous cell carcinoma (OSCC).

Materials and Methods: A total of 63 OSCC samples (21 samples each grade) were collected from the archive of Pathology Department of Besat educational hospital, Hamadan, Iran, from 2000 to 2015. VE-cadherin, CD44, and vimentin/periodic acid-Schiff (PAS) double-staining were used to validate VM. VM was identified by the detection of PAS-positive loops surrounded by tumor cells. Chi-square test was used to examine the differences between the variables. Significant level was set at 0.05 . Pearson's correlation was used to assess the co-localization of the markers.

Results: There were statistically significant differences between tumor grade and the expression levels of VE-cadherin, CD44, and vimentin $(P=0.000)$. In addition, significant differences were found between tumor grade and microvessel density $(P=0.000)$ and between tumor grade and VM $(P=0.000)$.

Conclusion: Our results may disclose a definite relationship between VE-cadherin, CD44 and vimentin expression levels, VM formation, EMT, CSCs, and microvessel count in OSCC samples. For this reason, it is suggested that VE-cadherin, CD44, and vimentin are related to angiogenesis and VM formation in OSCC, therefore, in tumor progression and metastasis. Recently, antitumor angiogenic therapies have been challenged. The presence of VM may explain the failure of antiangiogenic treatments.

KeYwords: CD44, oral squamous cell carcinoma, vasculogenic mimicry, vascular endothelial-cadherin, vimentin

\section{INTRODUCTION}

T ead and neck cancer is the sixth most common - cancer in the world..$^{[1]}$ Ninety percent of head and neck cancers are squamous cell carcinoma which develops in the oral cavity. ${ }^{[2,3]}$ Metastasis is the main causative factor for death in $90 \%$ of all cancer-related deaths ${ }^{[4-6]}$

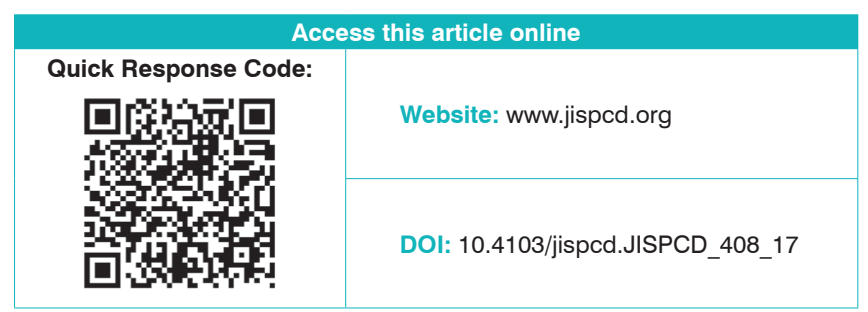

and begins with the invasion of tumor cells through the walls of small blood vessels or lymph vessels. ${ }^{[7,8]}$ Then,

Address for correspondence: Dr. Soussan Irani, Dental Faculty, Shahid Fahmideh Street, Hamadan, Iran. E-mail: sousanirani@gmail.com

This is an open access journal, and articles are distributed under the terms of the Creative Commons Attribution-NonCommercial-ShareAlike 4.0 License, which allows others to remix, tweak, and build upon the work non-commercially, as long as appropriate credit is given and the new creations are licensed under the identical terms.

For reprints contact: reprints@medknow.com

How to cite this article: Irani S, Dehghan A. The expression and functional significance of vascular endothelial-cadherin, CD44, and vimentin in oral squamous cell carcinoma. J Int Soc Prevent Communit Dent 2018;8:110-7. 
cancer cells settle into a niche to promote proliferation and vasculogenesis. ${ }^{[7,9]}$ Folkman first proposed a theory regarding tumor angiogenesis. According to this theory, a tumor forms new vasculature from existing blood vessels. ${ }^{[10]}$ Maniotis et al. indicated that the vascular-like channels which function as tumor blood vessels were formed in melanoma. This phenomenon was called "vasculogenic mimicry" (VM). ${ }^{[1]}$ A growing body of evidence has shown that VM formation facilitates tumor growth and cancer metastasis. ${ }^{[12]}$ Previously published works have shown that VM indicates a poor prognosis in oral squamous cell carcinoma (OSCC). ${ }^{[13]}$ Vascular endothelial-cadherin (VE-cadherin), an adhesive protein, promotes cell-to-cell interaction. Recently, VE-cadherin has been demonstrated in both endothelial cells and highly aggressive melanoma cells. ${ }^{[14]}$ Overexpression level of VE-cadherin enhances the cancer neovascularization, growth, and progression. ${ }^{[15,16]}$

On the other hand, a small subset of tumor cells involves in cancer development. These cells, cancer stem cells (CSCs), not only are able to reproduce the whole phenotype of the original tumor but also are capable of self-renewal. ${ }^{[17]}$ It is suggested that CSCs may also be involved in vascular formation in cancers. ${ }^{[18]}$ Previous publications have shown that CSCs can induce epithelial-mesenchymal transition (EMT) to promote tumor cell invasion and metastasis. ${ }^{[19]}$ CD44, a cell-surface glycoprotein, involves in cell-cell interactions, cell migration, and adhesion. For this reason, it plays a major role in tumorigenesis and metastasis. ${ }^{[20]}$ Furthermore, CD44 has been described as a CSC marker in head and neck squamous cell carcinoma (HNSCC) and can re-establish the tumor heterogeneity. ${ }^{[21]}$ Increased CD44 expression level has been detected in cancers such as HNSCC. ${ }^{[22]}$ There are controversial results regarding to CD44 expression level in OSCC and lymph node metastasis. While Fonseca et al, and Lindquist et al. indicated a positive relationship between high CD44 expression and lymph-node metastasis, ${ }^{[23,24]}$ Mostaan et al., and Rodrigo et al. found a correlation between low CD44 expression and capability for metastasis. ${ }^{[25,26]}$

Vimentin, a mesenchymal marker, plays a crucial role in EMT. ${ }^{[16,27]}$ In some cancers such as esophageal squamous cell carcinoma, the increased expression level of vimentin is associated with a higher incidence of lymph node metastasis. ${ }^{[28,29]}$ Recently, studies have shown that CSC and EMT enhance VM formation through stimulating cancer cell plasticity, remodeling the extracellular matrix, and connecting VM channels with host blood vessels, ${ }^{[30]}$ but the regulatory mechanism is still unclear. The identification of biomarkers related to EMT, CSCs, and VM may provide a chance to develop drugs targeting
EMT, CSC, and VM formation. ${ }^{[31,32]} \mathrm{VM}$ can be identified by the detection of periodic acid-Schiff (PAS)-positive loops surrounded by tumor cells (not endothelial cells), with or without red blood cells in it. ${ }^{[12]}$ The current study aimed to present the role of VE-cadherin, CD44, and vimentin in inducing $\mathrm{VM}$ and EMT and to identify the CSC niche in different grades of OSCC.

\section{Materialls and Methodos}

\section{PATIENTS AND TISSUE SAMPLES}

PASS software (power analysis and sample size) software (version 11.0.7; PASS, NCSS, LLC) was used to calculate the sample size using the following information: $\mathrm{DF}=4$, effect size $=0.5$, power $(1-\beta)=0.9$, and alpha (significance level $)=0.05$.

A total of 63 OSCC samples (21 samples each grade) were collected from the archive of Pathology Department of Besat Educational Hospital, Hamadan, Iran, from 2000 to 2015. Institutional Review Board approval number was 9409034804 .

There were 25 cases from the tongue, 15 cases from the buccal mucosa and 13 cases from gingiva, and 10 cases from floor of mouth. Adjacent normal oral mucosa served as control group. Hematoxylin and eosin staining was performed to confirm the previous diagnosis. Histologically, OSCCs were classified as low, intermediate, or high grade on the basis of the presence of cytological atypia, keratin pearl, mitotic activity, and other criteria. ${ }^{[33]}$

\section{DOUBLEIMIMUNOHISTOCHEMISTRY/PERIODICACID-SCHIFF STAINING}

The specimens were processed for immunohistochemistry analysis. Monoclonal anti-mouse IgG antibodies used in the immunohistochemistry assay were vimentin (Novocastra $^{\mathrm{TM}}$ ready to use) and CD44 (1:200; Thermo Scientific, Std./HCAM Ab-4). Polyclonal anti-rabbit VE-cadherin antibody (1:170; Abcam; 33168) was used as well. Then, the sections were stained with PAS. Briefly, tissue sections were cut by $4 \mathrm{~mm}$ thickness. Then, the sections were deparaffinized and dehydrated with graded alcohol. The antigen retrieval was done in citrate buffer $(\mathrm{pH}=6)$ for $\mathrm{CD} 44$ and vimentin and in EDTA/Tris $(\mathrm{pH}=9)$ for VE-cadherin. Using Leica detection kit, endogenous peroxidase activity was blocked. After 3 washes in Tris-buffered saline (TBS), the samples were incubated with primary antibodies for $1 \mathrm{~h}$. Negative controls were prepared by omitting the primary antibody. The positive control staining was also performed (reactive lymph node according to the manufacturer instructions). After TBS washing, the slides were developed in freshly prepared diaminobenzidine solution for $6 \mathrm{~min}$. Then, PAS staining was performed, 
followed by counterstaining with hematoxylin, dehydration, and mounting.

\section{DETECTION AND SCORING}

VE-cadherin and CD44 expression was detected in the membrane of the tumor cells. Vimentin expression was detected in the cytoplasm of cancer cells. Microvessel density (MVD) was determined by light microscopy examination of stained sections at the "hot spot." Fields of the greatest neovascularization were identified by light microscope at low power $(\times 100)$. The average vessel count of the five fields $(\times 400)$ was regarded as the MVD count. The MVD was classified as either high $(\geq 15)$ or low $(<15)$; 15 was considered as the median value of MVD in our study. VM was also assessed. VE-cadherin, CD44, and vimentin/PAS double-staining were used to validate VM. VM was identified by the detection of PAS-positive loops surrounded by tumor cells (not endothelial cells), with or without red blood cells in it. The abundance of positive cells for biomarkers was graded as follows: 1 (weak) for $<20 \%$ positive cells, 2 (moderate) for $20 \%-50 \%$ positive cells, and 3 (strong) for $>50 \%$ positive cells. ${ }^{[16]}$

\section{STATISTICAL ANALYSIS}

Analyses were conducted through SPSS software version 22.0 (SPSS, Inc., Chicago, IL, USA). Chi-Square test was used to examine the differences between the variables. Significant level was set at 0.05 . Pearson's

\begin{tabular}{|c|c|c|c|c|}
\hline $\begin{array}{l}\text { Histopathological } \\
\text { variables }\end{array}$ & Low grade & $\begin{array}{l}\text { Intermediate } \\
\text { grade }\end{array}$ & & $P$ \\
\hline \multicolumn{5}{|l|}{ VE-cadherin } \\
\hline Weak & $8(38.1 \%)$ & $12(57.2 \%)$ & 0 & $0.000 *$ \\
\hline Moderate & 0 & $7(33.3 \%)$ & $3(4.1 \%)$ & \\
\hline Strong & 0 & 0 & $18(24.3 \%)$ & \\
\hline Negative & $13(61.9 \%)$ & $2(9.5 \%)$ & 0 & \\
\hline \multicolumn{5}{|l|}{ CD44 } \\
\hline Weak & 0 & 0 & 0 & $0.000 *$ \\
\hline Moderate & $18(85.7 \%)$ & $7(33.3 \%)$ & 0 & \\
\hline Strong & $3(14.3 \%)$ & $14(66.7 \%)$ & $21(100 \%)$ & \\
\hline \multicolumn{5}{|l|}{ Vimentin } \\
\hline Weak & $13(61.9 \%)$ & 0 & 0 & $0.000 *$ \\
\hline Moderate & 0 & $18(85.7 \%)$ & $3(14.3 \%)$ & \\
\hline Strong & 0 & $3(14.3 \%)$ & $18(85.7 \%)$ & \\
\hline Negative & $8(38.1 \%)$ & 0 & 0 & \\
\hline \multicolumn{5}{|l|}{ MVD } \\
\hline$>15$ & 0 & $21(100 \%)$ & $21(100 \%)$ & $0.000 *$ \\
\hline$\leq 15$ & $21(100 \%)$ & 0 & 0 & \\
\hline \multicolumn{5}{|l|}{ VM } \\
\hline Positive & $6(28.6 \%)$ & $17(81.0 \%)$ & $18(85.7 \%)$ & $0.000^{*}$ \\
\hline Negative & $15(71.4 \%)$ & $4(19.0 \%)$ & $3(14.3 \%)$ & \\
\hline
\end{tabular}

correlation was used to assess the co-localization of the markers.

\section{RESULTS}

A total of 63 samples (40 men; $63.5 \%$, and 23 women; $36.5 \%$ ) were used for immunohistochemical study. Age ranged from 20 to 70 years with a mean age of 53.3 years. There were statistically significant differences between tumor grade and the expression levels of VE-cadherin, CD44, and vimentin $(P=0.000)$. In addition, significant differences were found between tumor grade and MVD $(P=0.000)$ and between tumor grade and VM $(P=0.000)$. Besides, there was a positive correlation between tumor grade and VEcadherin expression level Pearson $r=0.925, P<$ 0.000 ) between tumor grade and CD44 expression level (Pearson $r=0.595, P<0.000$ ), and between tumor grade and vimentin expression level (Pearson $r=0.678$, $P<0.000)$. The details are summarized in Table 1 .

\section{DISCUSSION}

In this study, the expression level of VE-cadherin, CD44, and vimentin was examined in different histological grades of OSCC. According to the previous studies, VM formation is seen involving in tumor growth and cancer metastasis, ${ }^{[11]}$ and in OSCC, it is correlated to poor prognosis. ${ }^{[9]}$ In addition, the elevated expression level of VE-cadherin is associated with the cancer growth and progression. ${ }^{[16]}$ Besides, VE-cadherin is expressed by CSCs and is associated with VM. ${ }^{[34,35]}$ A study on aggressive melanoma indicated the high expression level of VE-cadherin by cancer cells. The authors suggested that VE-cadherin expression by tumor cells enhances vasculogenic-like network formation. Furthermore, it is suggested that tumor plasticity allows VM formation which is correlated to the VE-cadherin expression. ${ }^{[14]}$ $\mathrm{VM}$ formation has a crucial role in the tumor progression and metastasis. ${ }^{[18]}$ Overexpression of VE-cadherin in cancers such as melanoma and breast cancer is associated with poor prognosis. ${ }^{[34]}$ In a published work, Fry et al. suggested VE-cadherin expression as a metastatic biomarker in breast cancer. ${ }^{[35]} \mathrm{VM}$ and EMT promote invasion and metastasis. ${ }^{[36,37,38]}$ During VM formation, highly aggressive epithelial tumor cells can overexpress the mesenchymal phenotype through EMT. ${ }^{[39]} \mathrm{VM}$ has been shown to present in 21/84 (25\%) of gastrointestinal stromal tumors, which were significantly associated with tumor grade and liver metastasis. ${ }^{[38]}$ In a previous study on OSCC, tumor cell-lined vessels were found in $18 / 33(54.5 \%)$ of cases. ${ }^{[13]}$ Besides, VM formation was found in $40 \%$ of adenoid cystic carcinoma (ACC) tissues, mainly in the solid pattern. ${ }^{[19]}$ Importantly, a published work on the triple-negative 
breast cancer demonstrated a significant expression level of VE-cadherin in CD133+ CSCs. The authors proposed that CD133+ CSCs might have the ability of acquisition of endothelial cell phenotype and VE-cadherin expression to enhance VM formation. ${ }^{[39]}$ In our study, strong expression level of VE-cadherin was found in all high-grade samples. In addition, VM formation (lined by VE-cadherin-positive cancer cells) was detected in $17(81 \%)$ of intermediate-grade and $18(85.7 \%)$ of high-grade samples [Figures 1 and 2]. Furthermore, VM channels lined by VE-cadherin-positive cancer cells were mostly detected in histologically higher degree samples and the VE-cadherin expression level increased by the increasing of tumor grade increase. The present study also demonstrated VE-cadherin positivity at invasive front [Figure 3]. These results may provide sufficient document for the role of VE-cadherin expression level and VM formation in the tumor growth and the risk of metastasis through CSCs and EMT. Moreover, VE-cadherin expression level is suggested as a metastatic biomarker for OSCC.

Regarding CD44 expression level in the present study, strong CD44 expression was found in all high-grade samples and $13(61.9 \%)$ of intermediate-grade samples [Figures 4 and 5]. Besides, CD44-positive cancer cells were mainly found at invasive front and at the periphery of tumor islands [Figure 6]. Therefore, the present study provides enough evidence that the number of CSCs also increases by the increase of histological degree, and also, CD44 and VE-cadherin enhance tumor growth and metastasis by increasing the number of CSCs. CD44 expression is correlated with CSCs, and the aggressiveness of head and neck tumors $^{[22]}$ and CD44 plays a critical role in VE-cadherin

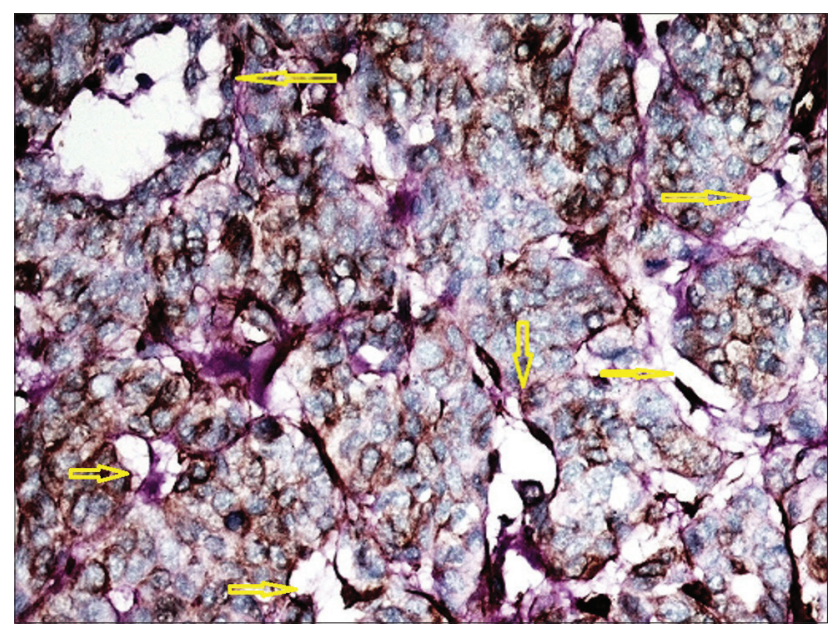

Figure 1: Histologic section of intermediate-grade tumor shows a strong vascular endothelial-cadherin positivity. Yellow arrows indicate vascular channels lined by vascular endothelial-cadherin-positive tumor cells $(\times 400)$ expression. ${ }^{[40]}$ For instance, a previously published paper indicated that CD44 plays an important role in controlling the proliferation and apoptosis of capillary endothelial cells through CD31 and VE-cadherin expression. ${ }^{[40]}$ A published study demonstrated that CD44 variant mediates disassembly of endothelial VE-cadherin junction on metastatic melanoma cells. By doing this, CD44 mediates melanoma cell transendothelial migration. ${ }^{[41]}$ CD44 is expressed in the basal cell layer of normal oral mucosa as well as $\mathrm{CSCs}^{[21,42]} \mathrm{A}$ previous study on HNSCC samples found CD44 expression in all samples tested. ${ }^{[22]}$ However, another published study on different histologic grades of OSCC indicated CD44 positivity in $80 \%-100 \%$ of well-differentiated cases, in $60 \%-85 \%$ of moderate-differentiated samples, and in $40 \%-62 \%$ of poor-differentiated cases. The reaction was mostly found at the periphery of tumor islands. ${ }^{[23]}$

According to the results obtained in the current investigation, elevated expression of vimentin was also indicated in tumor cells mainly at the periphery of tumor islands and invasive front in intermediate- and high-grade samples [Figures 7 and 8]. Moreover, vimentin positivity was found in some detached cancer cells, especially around the blood vessels, and in the stroma indicating the tumor cells which acquired EMT properties [Figure 9]. Previous studies on HNSCC samples, strong positivity of vimentin was found in the microenvironment. The authors proposed that cancer cell mobility enhances cancer progression and metastasis. ${ }^{[43,44]}$ In other cancers such as colon, breast, and prostate cancers, vimentin expression level was indicative of aggressive cancer behavior and poor prognosis. ${ }^{[45,46]}$ Recently published work has shown that elevated vimentin expression level is correlated with EMT process of cancers such as

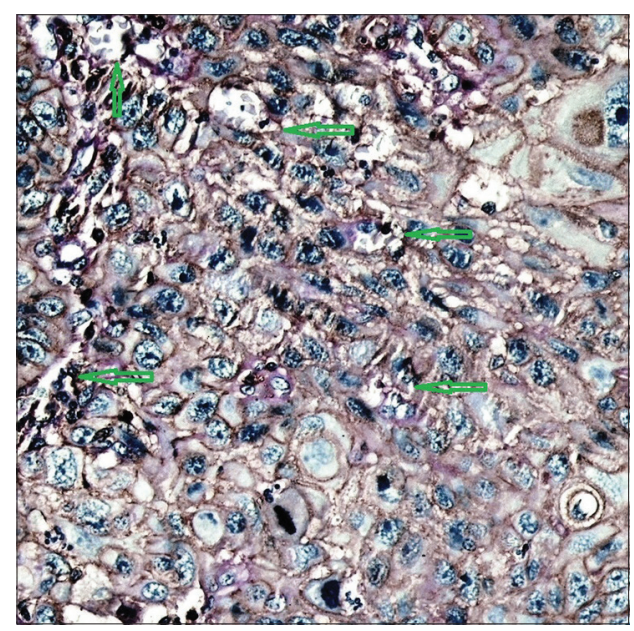

Figure 2: Paraffin section of high-grade tumor. The high-power magnification view shows a strong vascular endothelial-cadherin cell membrane staining of undifferentiated tumor cells. Green arrows indicate vascular channels lined by vascular endothelial-cadherin tumor cells 


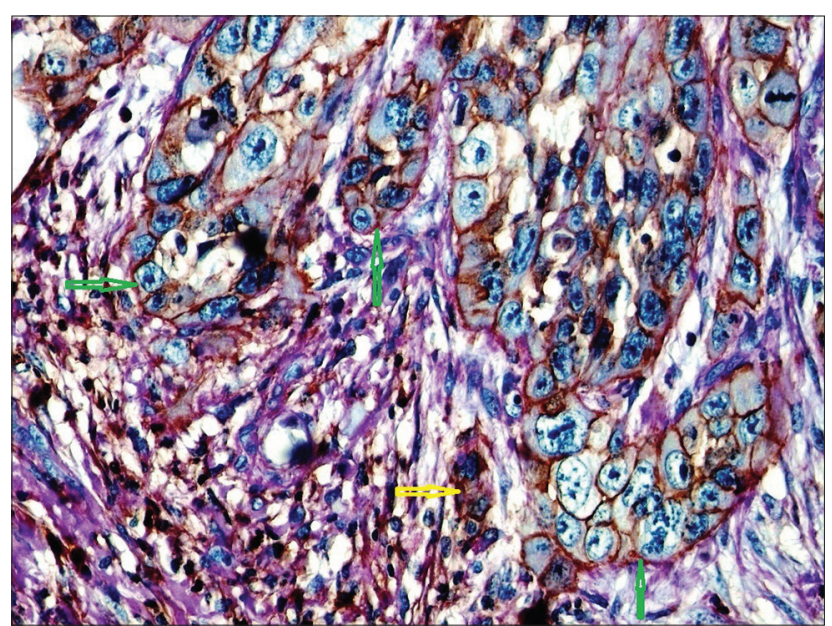

Figure 3: Formalin-fixed, paraffin-embedded tissue section indicating a strong vascular endothelial-cadherin positivity at invasive front (green arrows). Yellow arrow shows vascular endothelial-cadherin positivity in some detached tumor cells $(\times 400)$

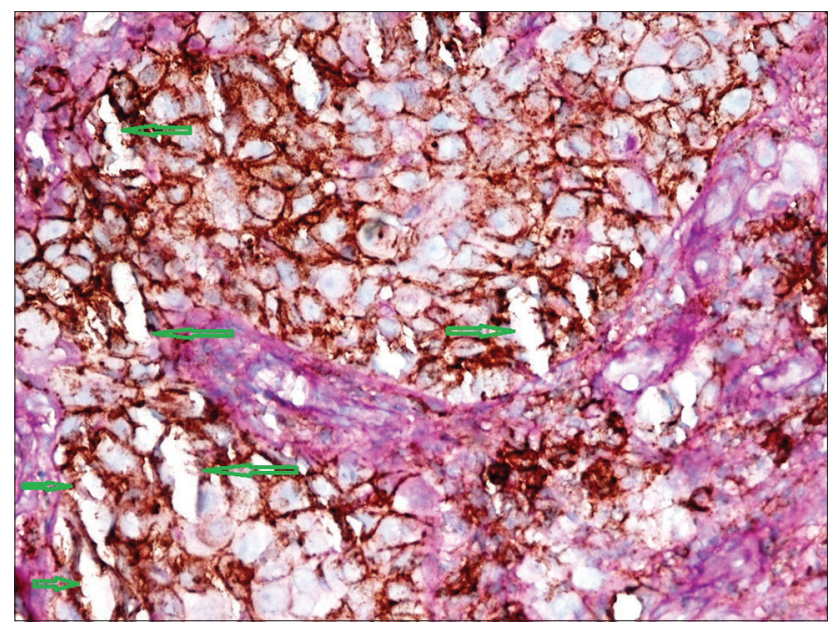

Figure 5: High-power section of intermediate-grade tumor demonstrates a strong CD44 positivity in tumor cells. Green arrows indicate vascular channels lined by CD44-positive tumor cells

breast cancer ${ }^{[47]}$ and is indicative of poor prognosis in OSCC. ${ }^{[44]}$ Furthermore, vimentin expression is associated with esophageal squamous cell carcinoma lymph node metastasis. ${ }^{[28,29]}$ EMT in an epithelial tumor may be an alternative mechanism of VM formation. ${ }^{[38]}$ On the other hand, it is suggested that CSCs may be involved in vascular formation ${ }^{[13]}$ and EMT to promote tumor cell invasion and metastasis. ${ }^{[14]}$ The current study provides enough evidence that the higher expression levels of CD44 and vimentin at the periphery of tumor islands and invasive front mostly in higher grade samples may indicate that CSC properties are necessary to get EMT properties which, in turn, enhance the cancer metastasis. Taken together, in the current study, some tumor cells especially at the periphery of tumor islands and at invasive front got stained with VE-cadherin, CD44, and vimentin showing that they are CSCs which could get EMT

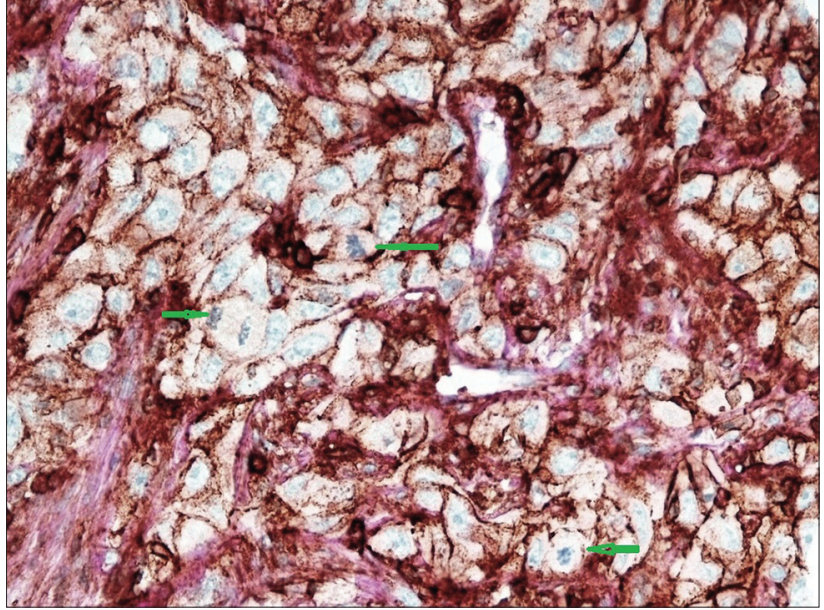

Figure 4: High-power section of high-grade tumor demonstrates a strong CD44 positivity in tumor cells. Green arrows indicate the mitotic figures

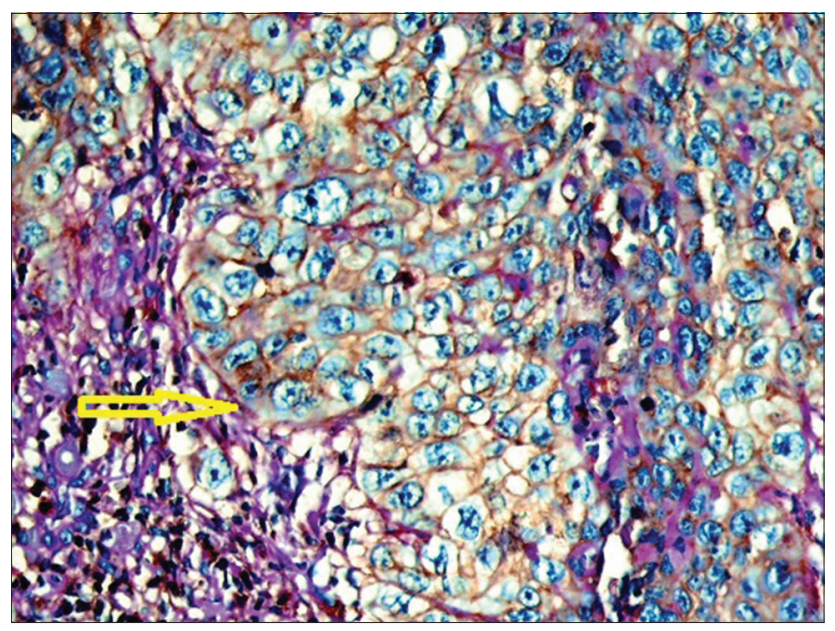

Figure 6: High power of intermediate-grade sample indicates CD44 positivity in all tumor cells. Yellow arrow indicates the tumor-stroma interface

phenotype. In addition, it can be suggested that these cells are involved in VM formation. Collectively, the current study can be another proof for the previous hypotheses regarding the role of CSC, EMT, and VM channels in cancer development and metastasis. Furthermore, the present study provides enough information about some other molecules and pathways in the cancer growth and metastasis in OSCC. More information about the involved molecules and pathways in tumor growth, especially in metastasis can help to design future investigations to provide new drugs to prevent metastasis as the most important reason for death in oral cancer patients.

MVD is another key player in tumor behavior. In the present study, MVD count was higher in intermediate- and high-grade samples compared to that of low-grade cases. Similar to the current study, a previous study on prostate cancer found a significant association between microvessel count (MVC) and tumor grade. ${ }^{[46]}$ 


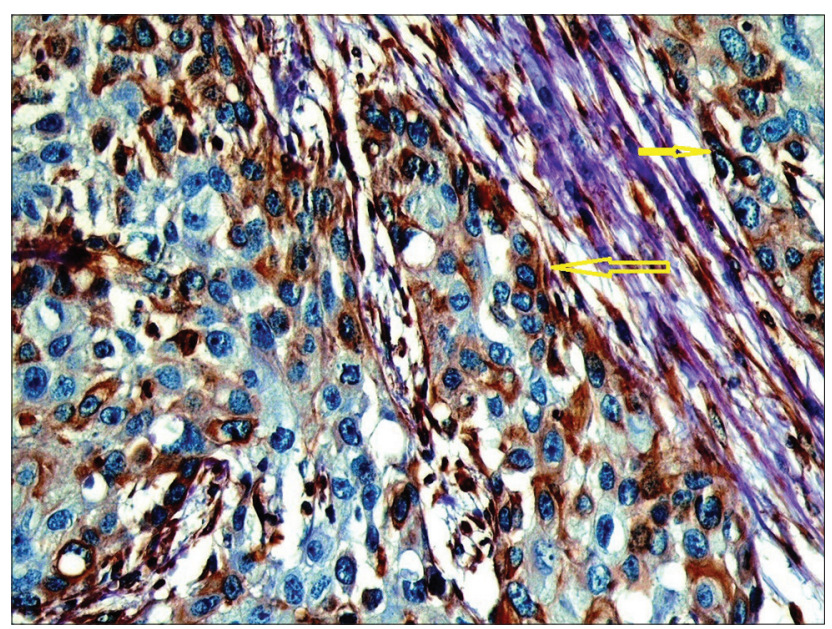

Figure 7: Paraffin section of intermediate grade tumor. The high-power magnification view shows a strong vimentin cytoplasmic staining of tumor cells mainly at the periphery of tumor islands (yellow arrows)

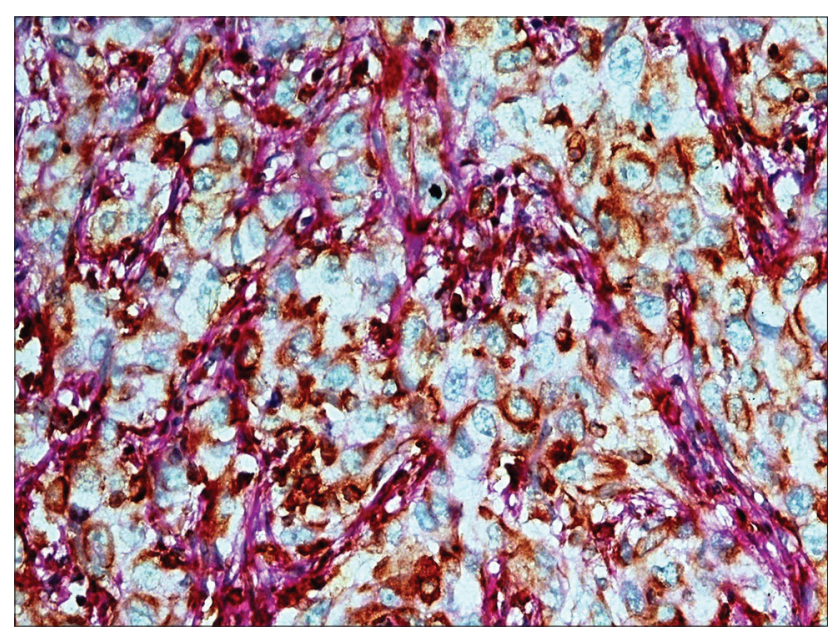

Figure 8: High-power photomicrograph from high-grade oral squamous cell carcinoma. Note the cytoplasmic positivity of vimentin in tumor cells

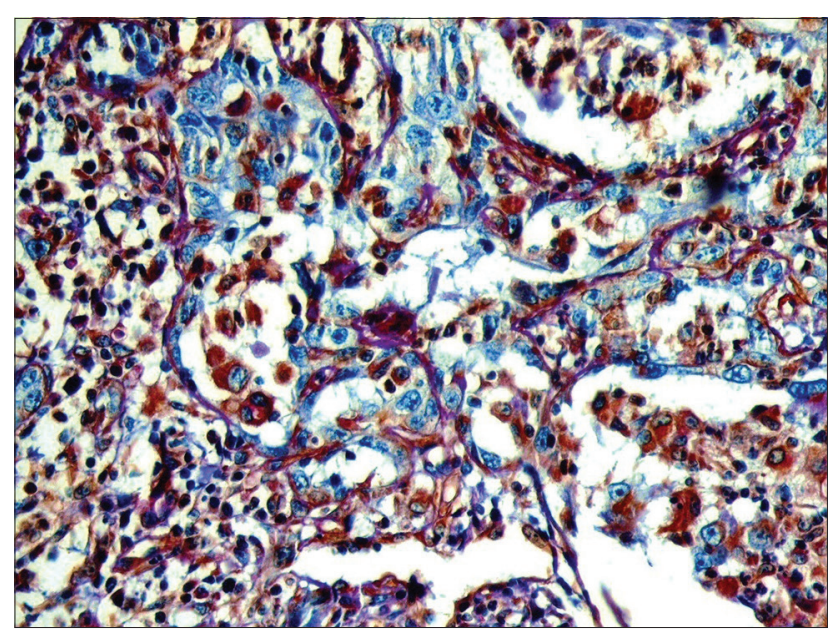

Figure 9: High-power magnification shows cytoplasmic vimentin positivity in detached tumor cells and stromal cells

In ACC samples, MVD was correlated significantly with the clinical stage, vascular invasion, and metastasis. ${ }^{[48]}$

\section{Conclusion}

Our results may disclose a definite relationship between VE-cadherin, CD44 and vimentin expression levels, VM formation, EMT, CSCs, and MVC in OSCC samples. For this reason, it is suggested that VE-cadherin, CD44, and vimentin are related to angiogenesis and VM formation in OSCC, therefore, in tumor progression and metastasis. Recently, antitumor angiogenic therapies have been challenged. The presence of VM may explain the failure of antiangiogenic treatments. ${ }^{[18]}$ Thus, the inhibition of VM formation has become a new strategy for anticancer therapy. Besides, mutations due to a genetic instability and environmental factors make the solid tumors such as OSCC heterogeneous. ${ }^{[4,50]}$ Heterogeneity enhances tumor formation which may prove CSC hypothesis. ${ }^{[50]}$ It is suggested that these cells give the tumor the ability to be resistant to chemoradiotherapy and metastasize. ${ }^{[51]}$ Identifying the biomarkers of stem cells which acquire EMT characteristics may also improve the development of drugs targeting EMT CSCs. ${ }^{[52-55]}$

\section{ACKNOWLEDGMENT}

The authors would like to acknowledge the funding from Hamadan University of Medical Sciences.

\section{FINANCIAL SUPPORT AND SPONSORSHIP}

This study was financially supported by Hamadan University of Medical Sciences.

\section{CONFLICTS OF INTEREST}

There are no conflicts of interest.

\section{REFERENCES}

1. Parkin DM, Bray F, Ferlay J, Pisani P. Global cancer statistics, 2002. CA: Cancer J Clin 2005;55:74-108.

2. Warnakulasuriya S. Global epidemiology of oral and oropharyngeal cancer. Oral Oncol. 2009;45:309-16.

3. Irani S, Bidari Zerehposh F, Sabeti Sh. Prevalence of Pathological Entities in Neck Masses: A Study of 1208 Consecutive Cases. Avicenna J Dent Res. 2016;8:1-5.

4. Spano D, Heck C, De Antonellis P, Christofori G, Zollo M. Molecular networks that regulate cancer metastasis. Semin Cancer Biol 2012;22:234-49.

5. Irani S. Metastasis to the oral soft tissues: A review of 412 cases. J Int Soc Prev Community Dent 2016;6:393-401.

6. Irani S. Metastasis to the Jawbones: A review of 453 cases. J Int Soc Prev Community Dent 2017;7:71-81.

7. Irani S. Metastasis to head and neck area: a 16-year retrospective study. Am J Otolaryngol 2011;32:24-7.

8. Irani S. Pre-Cancerous Lesions in the Oral and Maxillofacial Region: A Literature Review with Special Focus on Etiopathogenesis. Iran J Pathol 2016;11:303-22.

9. Irani S. Distant metastasis from oral cancer: A review and molecular biologic aspects. J Int Soc Prev Community Dent 2016;6:265-71.

10. Folkman J. Angiogenesis: an organizing principle for drug discovery? Nat Rev Drug Discov 2007;6:273-86.

11. Maniotis AJ, Folberg R, Hess A, Seftor EA, Gardner LM, Pe'er J, 
et al. Vascular channel formation by human melanoma cells in vivo and in vitro: vasculogenic mimicry. Am J Pathol 1999;155:739-52.

12. Wang W, Lin P, Han C, Cai W, Zhao X, Sun B. Vasculogenic mimicry contributes to lymph node metastasis of laryngeal squamous cell carcinoma. J Exp Clin Cancer Res: CR. 2010;29:60.

13. Liu SY, Chang LC, Pan LF, Hung YJ, Lee CH, Shieh YS. Clinicopathologic significance of tumor cell-lined vessel and microenvironment in oral squamous cell carcinoma. Oral Oncol 2008;4:277-85.

14. Hendrix MJ, Seftor EA, Meltzer PS, Gardner LM, Hess AR, Kirschmann DA, et al. Expression and functional significance of VEcadherin in aggressive human melanoma cells: role in vasculogenic mimicry. Proc Natl Acad Sci U S A 2001;98:8018-23.

15. Azzi S, Hebda JK, Gavard J. Vascular permeability and drug delivery in cancers. Front Oncol 2013;3:211.

16. Irani S, Dehghan A. Expression of VE-Cadherin in Mucoepidermoid Carcinoma: Role in cancer development. J Int Soc Prev Community Dent 2017;7:301-7.

17. Lee HJ, Kang YH, Lee JS, Byun JH, Kim UK, Jang SJ, et al. Positive expression of NANOG, mutant p53, and CD44 is directly associated with clinicopathological features and poor prognosis of oral squamous cell carcinoma. BMC oral health. 2015;15:153.

18. Zhang Y, Sun B, Zhao X, Liu Z, Wang X, Yao X, et al. Clinical significances and prognostic value of cancer stem-like cells markers and vasculogenic mimicry in renal cell carcinoma. J Surg Oncol 2013;108:414-9.

19. Wang SS, Gao XL, Liu X, Gao SY, Fan YL, Jiang YP, et al. CD133+ cancer stem-like cells promote migration and invasion of salivary adenoid cystic carcinoma by inducing vasculogenic mimicry formation. Oncotarget. 2016.

20. Mannelli G, Gallo O. Cancer stem cells hypothesis and stem cells in head and neck cancers. Cancer Treat Rev 2012;38:515-39.

21. Prince ME, Sivanandan R, Kaczorowski A, Wolf GT, Kaplan MJ, Dalerba $\mathrm{P}$, et al. Identification of a subpopulation of cells with cancer stem cell properties in head and neck squamous cell carcinoma. Proc Natl Acad Sci U S A 2007;104:973-8.

22. Joshua B, Kaplan MJ, Doweck I, Pai R, Weissman IL, Prince ME, et al. Frequency of cells expressing CD44, a head and neck cancer stem cell marker: correlation with tumor aggressiveness. Head Neck 2012;34:42-9.

23. Fonseca I, Pereira T, Rosa-Santos J, Soares J. Expression of CD44 isoforms in squamous cell carcinoma of the border of the tongue: A correlation with histological grade, pattern of stromal invasion, and cell differentiation. J Surg Oncol 2001;76:115-20.

24. Lindquist $\mathrm{D}$, Ahrlund-Richter $\mathrm{A}$, Tarjan $\mathrm{M}$, Tot $\mathrm{T}$, Dalianis $\mathrm{T}$. Intense CD44 expression is a negative prognostic factor in tonsillar and base of tongue cancer. Anticancer research. 2012;32:153-61.

25. Mostaan LV, Khorsandi MT, Sharifian SM, Shandiz FH, Mirashrafi F, Sabzari H, et al. Correlation between E-cadherin and CD44 adhesion molecules expression and cervical lymph node metastasis in oral tongue SCC: Predictive significance or not. Pathol Res Pract 2011;207:448-51.

26. Rodrigo JP, Dominguez F, Alvarez C, Herrero A, Suarez C. Expression of E-cadherin, CD44s, and CD44v6 in laryngeal and pharyngeal carcinomas. Am J Otolaryngol. 2003;24:384-9.

27. Luo T, Wang $\mathrm{L}$, Wu $\mathrm{P}$, Gong $\mathrm{W}$, Chen $\mathrm{W}$, Zhao $\mathrm{H}$, et al. Downregulated vimentin and upregulated E-cadherin in T1 stage non-small-cell lung cancer: does it suggest a mesenchymal-epithelial transition? Neoplasma 2017.

28. Irani S, Salajegheh A, Smith RA, Lam AK. A review of the profile of endothelin axis in cancer and its management. Crit Rev Oncol Hematol. 2014;89:314-21.

29. Irani S, Salajegheh A, Gopalan V, Smith RA, Lam AK. Expression profile of endothelin 1 and its receptor endothelin receptor A in papillary thyroid carcinoma and their correlations with clinicopathologic characteristics. Ann Diagn Pathol 2014;18:43-8.
30. Sun B, Zhang D, Zhao N, Zhao X. Epithelial-to-endothelial transition and cancer stem cells: two cornerstones of vasculogenic mimicry in malignant tumors. Oncotarget 2017;8:30502-10.

31. Yang J, Yang Q, Yu S, Zhang X. Endocan: A new marker for cancer and a target for cancer therapy. Biomed Rep 2015;3:279-83.

32. Huang GW, Tao YM, Ding X. Endocan expression correlated with poor survival in human hepatocellular carcinoma. Dig Dis Sci 2009;54:389-94.

33. Neville B DD, Allen C, Chi A. Oral and Maxillofacial Pathology. $4^{\text {th }}$ ed. China: Elsevier; 2016.

34. Bartolome RA, Torres S, Isern de Val S, Escudero-Paniagua B, Calvino E, Teixido J, et al. VE-cadherin RGD motifs promote metastasis and constitute a potential therapeutic target in melanoma and breast cancers. Oncotarget. 2017;8:215-27.

35. Fry SA, Sinclair J, Timms JF, Leathem AJ, Dwek MV. A targeted glycoproteomic approach identifies cadherin-5 as a novel biomarker of metastatic breast cancer. Cancer Lett 2013;328:335-44.

36. Wang W, Lin P, Sun B, Zhang S, Cai W, Han C, et al. Epithelialmesenchymal transition regulated by EphA2 contributes to vasculogenic mimicry formation of head and neck squamous cell carcinoma. Biomed Res Int 2014;2014:803914.

37. Liu Q, Qiao L, Liang N, Xie J, Zhang J, Deng G, et al. The relationship between vasculogenic mimicry and epithelialmesenchymal transitions. Journal of cellular and molecular medicine. 2016;20:1761-9.

38. Sun B Qie S, Zhang S, Sun T, Zhao X, Gao S. Role and mechanism of vasculogenic mimicry in gastrointestinal stromal tumors. Hum Pathol 2008;39:444-51.

39. Liu TJ, Sun BC, Zhao XL, Zhao XM, Sun $\mathrm{T}, \mathrm{Gu} \mathrm{Q}$, et al. CD133+ cells with cancer stem cell characteristics associates with vasculogenic mimicry in triple-negative breast cancer. Oncogene. 2013;32:544-53.

40. Tsuneki M, Madri JA. CD44 regulation of endothelial cell proliferation and apoptosis via modulation of CD31 and VE-cadherin expression. J Biol Chem 2014;289:5357-70.

41. Zhang P, Fu C, Bai H, Song E, Dong C, Song Y. CD44 variant, but not standard CD44 isoforms, mediate disassembly of endothelial VE-cadherin junction on metastatic melanoma cells. FEBS letters. 2014;588:4573-82.

42. Jones KB, Klein OD. Oral epithelial stem cells in tissue maintenance and disease: the first steps in a long journey. Int $\mathrm{J}$ Oral Sci 2013;5:121-9.

43. Dutsch-Wicherek M. RCAS1, MT, and vimentin as potential markers of tumor microenvironment remodeling. Am J Reprod Immunol (New York, NY : 1989). 2010;63:181-8.

44. Chaw SY, Majeed AA, Dalley AJ, Chan A, Stein S, Farah CS Epithelial to mesenchymal transition (EMT) biomarkers--Ecadherin, beta-catenin, APC and Vimentin--in oral squamous cell carcinogenesis and transformation. Oral Oncol 2012;48:997-1006.

45. Cao Z, Shang B, Zhang G, Miele L, Sarkar FH, Wang Z, et al. Tumor cell-mediated neovascularization and lymphangiogenesis contrive tumor progression and cancer metastasis. Biochim Biophys Acta 2013;1836:273-86.

46. Dugonjic AS, Usaj SK, Eri Z, Latinovic LT. Significance of microvessel density in prostate cancer core biopsy. Vojnosanit Pregl 2015;72:317-27.

47. Liu T, Zhang X, Shang M, Zhang Y, Xia B, Niu M, Liu Y, Pang D. Dysregulated expression of Slug, vimentin, and E-cadherin correlates with poor clinical outcome in patients with basal-like breast cancer. J Surg Oncol. 2013;107:188-94.

48. Zhang J, Peng B, Chen X. Expressions of nuclear factor kappaB, inducible nitric oxide synthase, and vascular endothelial growth factor in adenoid cystic carcinoma of salivary glands: Correlations with the angiogenesis and clinical outcome. Clin Cancer Res 2005; 11:7334-43

49. Pai SI, Westra WH. Molecular pathology of head and neck cancer: implications for diagnosis, prognosis, and treatment. Annu Rev 
Pathol 2009;4:49-70.

50. Golub TR. Genome-wide views of cancer. $N$ Engl $J$ Med 2001;344:601-2.

51. Allegra E, Trapasso S. Cancer stem cells in head and neck cancer. Onco Targets Ther 2012;5:375-83.

52. Radisky DC, LaBarge MA. Epithelial-mesenchymal transition and the stem cell phenotype. Cell Stem Cell 2008;2:511-2.

53. Iwatsuki M, Mimori K, Yokobori T, Ishi H, Beppu T, Nakamori S, et al. Epithelial-mesenchymal transition in cancer development and its clinical significance. Cancer Sci 2010;101:293-9.

54. Albers AE, Chen C, Koberle B, Qian X, Klussmann JP, Wollenberg B, et al. Stem cells in squamous head and neck cancer Crit Rev Oncol Hematol 2012;81:224-40.

55. Irani S, Jafari B. Expression of vimentin and CD44 in Mucoepidermoid Carcinoma: A role in tumor growth. Indian J Den Res 2018. [In press]. 